\title{
23 CHALLENGING THE STATUS QUO: ENGAGING YOUTH IN THE DEVELOPMENT OF A CURRICULUM RESOURCE ABOUT ALCOHOL AND YOUNG PEOPLE
}

doi:10.1136/injuryprev-2012-040590o.23

\author{
${ }^{1}$ S Stuart, ${ }^{2} \mathrm{~L}$ Cvitanovic. ${ }^{7}$ ACC, New Zealand; ${ }^{2}$ Whakauae Research, New Zealand
}

Background Combating alcohol related harm in New Zealand takes a concerted effort by many sectors of the community working together with a shared vision. Needs analysis identified a dearth of youth alcohol harm minimisation resource material to support curriculum delivery in Whanganui secondary schools. The project aimed to co-ordinate development of a youth informed digital video disc (DVD) to address this gap. It sought to engage youth in conversations around harm minimisation and curriculum resource development and to increase community collaboration in support of this. Evaluation focussed on project activities and short term outcomes. Aims/Objectives/Purpose Evaluation aims included identifying levels of stakeholder engagement, particularly among youth, factors influencing engagement and impact of the resource on youth awareness.

Methods Mixed methods were used with a focus on a qualitative approach. Information was collected via debrief sessions, document analysis (including photographic records), youth pre and post DVD viewing feedback and participant observation.

Results/Outcomes Youth were successfully engaged in key project implementation phases. This was influenced by opportunities to have meaningful input. Youth pre and post DVD viewing data indicated significant increases in awareness of the effects of alcohol and of strategies for keeping safer around alcohol.

Significance/Contribution to the Field A youth informed DVD resource is now available to support alcohol harm minimisation curriculum delivery in Whanganui. The potential for the DVD to be used more widely has been identified with ACC developing complimentary 'wrap around' resources to encourage familial relationships and conversations about alcohol and young people. Communities can individualise the resource to suit their needs. 